\title{
Splenectomy for immune thrombocytopenia: the evolution and preservation of treatment
}

\author{
Allison Remiker ${ }^{1}$ and Cindy Neunert ${ }^{2}$ \\ ${ }^{1}$ Division of Pediatric Hematology, Oncology, Neuro-Oncology, and Stem Cell Transplantation, Ann \& Robert H. Lurie Children's \\ Hospital of Chicago, Northwestern University Feinberg School of Medicine, Chicago, IL and ${ }^{2}$ Division of Pediatric \\ Hematology/Oncology/Stem Cell Transplantation, Columbia University Medical Center, New York, NY, USA \\ E-mail: CINDYNEUNERT-cn2401@cumc.columbia.edu
}

doi:10.3324/haematol.2020.261099

T he management of immune thrombocytopenia (ITP) has evolved over the course of the past twenty-five years as new treatments have emerged (Figure 1). Despite such advances, splenectomy remains a viable choice. In this edition of Haematologica, Avila et al. provide long-term outcomes of pediatric patients with ITP who underwent splenectomy. Using data derived from the Splenectomy Registry of the Intercontinental Cooperative ITP Study (ICIS) group, an update of outcomes of patients with primary ITP who underwent splenectomy treatment between 1997 and 2017 was analyzed. ${ }^{1}$ Findings from this study provide important and relevant information regarding splenectomy as an effective treatment option for ITP, which in certain contexts may have improved outcomes.

Immune thrombocytopenia was first described even prior to the identification of platelets as a component of blood. Various conditions associated with purpura were described from the $11^{\text {th }}$ to the $17^{\text {th }}$ century., ${ }^{2,3}$ However, it was only in 1735 that Paul Gottleib Werlhof reported the first classical case of ITP4 in a teenage girl with cutaneous and overt mucosal bleeding symptoms following an infectious disease. This led to the eponym of "Werlhof's Disease", which was previously used to describe ITP. ${ }^{3}$ Over time, as microscopy technology progressed, platelets were discovered. Shortly after Bizzozero's discovery of the association between presence and function of platelets in 1881, our understanding of the pathophysiology of thrombocytopenia grew during the late $1880 \mathrm{~s} .{ }^{5}$

Hypotheses began to emerge regarding the pathophysiology of ITP, with respect to a state of either poor platelet production versus a process of peripheral platelet destruction. In the early 1900s, Marino inoculated guinea pigs with rabbit platelets producing antiplatelet antibodies, this simulated ITP in humans suggesting an immunemediated destructive cause. ${ }^{6}$ Years later, in 1916, Paul Kaznelson extrapolated the pathophysiology of immunemediated hemolytic anemia to ITP and suggested that platelet destruction occurred in the spleen. This led to the first successful splenectomy in a 36-year-old woman with presumed chronic ITP, improving her platelet count from $2 \times 10^{9} / \mathrm{L}$ to $500 \times 10^{9} / \mathrm{L} .^{78}$ Finally, in 1950 Dr. Harrington injected himself with blood from a woman with ITP; his platelet count immediately dropped, recovering five days later. This experiment was the first to support the concept of an anti-platelet factor in the blood. ${ }^{9}$ Over the course of the following hundred years, knowledge of the pathophysiology of ITP has continued to expand to embrace a comprehensive recognition of the complex interactions in the immune system, in turn leading to a variety of novel treatment modalities., ${ }^{2,3}$ Despite these advances, splenectomy has continued to stand the test of time since it was first performed in 1916, remaining a beneficial option for this condition.

Treatment guidelines for ITP were first established through the British Paediatric Haematology Group in 1992, soon followed by American Society of Hematology (ASH) guidelines in 1996. These initial guidelines outlined expert consensus-based practice standards for the evaluation and treatment of children and adults with ITP. ${ }^{10,11}$ Subsequently, revisions of the ASH guidelines were published in 2011, and most recently in late 2019 applying more rigorous evidence-based methodology. ${ }^{12,13}$ Recommendations for secondary treatment for primary ITP in the original guidelines were limited to splenectomy. There were minimal data on the use of splenectomy in children, with one study demonstrating a $72 \%$ rate of complete remission..$^{14}$ Evidence of adverse effects was also insufficient, and non-specific to ITP. Both early guidelines encouraged delaying splenectomy until children had had ITP for at least 12 months and also reserved splenectomy for children with bleeding symptoms. The ASH 1996 guidelines further suggested that patients have a platelet count $<10 \times 10^{9} / \mathrm{L}$ (ages 3-12 years), or 10 $30 \times 10^{9} / \mathrm{L}$ with bleeding symptoms (ages $8-12$ years). ${ }^{10}$

The next significant breakthrough in the treatment for ITP occurred with rituximab. Rituximab, a monoclonal CD20 antibody, was first used for the treatment of B-cell lymphomas in the 1980s. The first report of its use for autoimmune disease was published in 1998, and, in 2001, Stasi et al. reported on the first prospective trial of rituximab in adult patients with chronic ITP $(\mathrm{n}=25){ }^{15,16}$ It was not until 2006, however, that the first trial was conducted in children. ${ }^{17}$ With this new advent of successful non-surgical treatment, the 2011 ASH guidelines suggested the use of rituximab or high-dose dexamethasone as initial treatments for persistent, chronic or refractory children while it was suggested that splenectomy be used after other measures had failed, and again be delayed to at least 12 months of disease with persistence with bleeding symptoms or need for improved quality of life (HRQoL). ${ }^{12}$ The most recent advancement in the treatment of ITP has been the development of the thrombopoietin receptor agonists (TPO-RA) following recognition of impaired thrombopoiesis and megakaryocyte apoptosis in ITP patients. ${ }^{18,19}$ Both eltrombopag and romiplostim are now approved for children with persistent or chronic ITP who have insufficient response to corticosteroids, immunoglobulins, or splenectomy. The most recent ASH guidelines recommended both the use of TPO-RA and rituximab prior to splenectomy, based only on single arm prospective studies. The pooled evidence demonstrated a $91 \%$ response rate to splenectomy after one month, and a $77 \%$ durable response, which was 


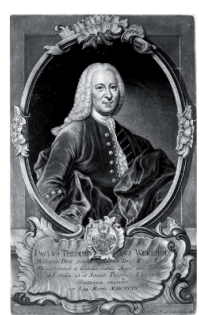

Idiopathic thrombocytopenic purpura Platelets 8 cases
thousands

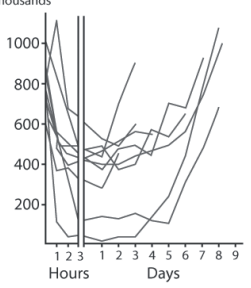

1735

First description of ITP (Werlhof Disease)

\section{0}

Established ITP as an autoimmune blood disorder

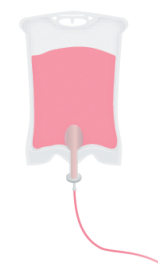

1998

First case report of rituximab

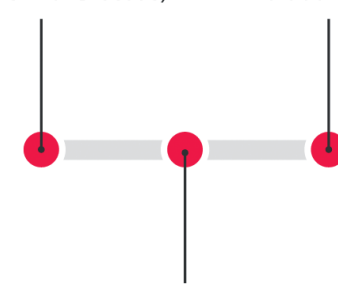

1916 First splenectomy conducted

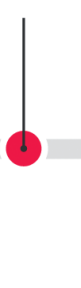

1994

Successful cloning of thrombopoietin

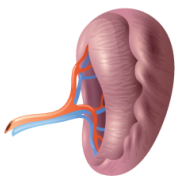

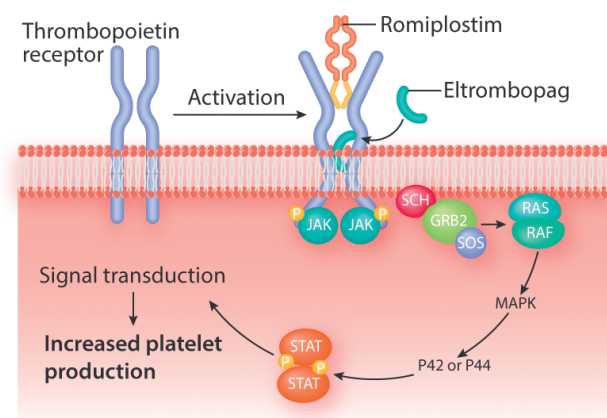

2006

First rituximab trial in pediatrics

\section{8 \\ Eltrombopag}

FDA approval

for adults
Romiplostim extended for children

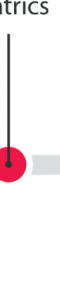

2003-2004

Confirmation of impaired

thrombopoiesis related to

platelet antibodies

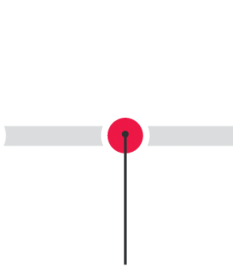

\section{8}

Romiplostim FDA approva for adults
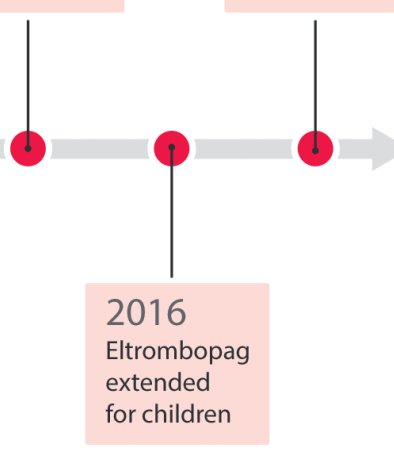

Figure 1. History of discovery and therapies for immune thrombocytopenia (ITP). FDA: US Food and Drug Administration.

superior to both alternative treatments. However, given the moderate undesirable effects associated with splenectomy, including fatal sepsis and lifelong susceptibility to bacterial infection, TPO-RA and rituximab were preferred. $^{13}$

With the decline in the number of splenectomies performed in children with ITP, despite potential data supporting superior efficacy to alternative therapies and guidelines favoring newer more novel therapies, Avila et al. used the ICIS Splenectomy Registry to evaluate longterm outcomes in 239 children with ITP. ${ }^{1}$ Analysis duration was a median of 25 months and included assessments for response and bleeding events. Interestingly, $26 \%$ of patients had splenectomies performed in the acute or persistent phase of diagnosis, which is outside the commonly recommended window per guidelines. Minimal adverse events occurred in the peri-operative window with $5 \%$ having intra-abdominal bleeding, $10 \%$ with fever, and no deaths or reports of sepsis. Of the patients followed for $\geq 6$ months $(n=168), 11 \%$ had admissions for fever and $2.7 \%$ for sepsis. Response was notable for $93 \%$ of patients achieving complete remission (CR: $\geq 60 \%$ of platelet counts $\geq 1$ month post splenectomy $\geq 100 \times 10^{\%} / \mathrm{L}$ ) or remission ( $\mathrm{R}: \geq 60 \%$ of platelet counts $\geq 1$ month post splenectomy $\geq 30 \times 10^{\%} / \mathrm{L}$ ). Refractoriness was seen in only $1.7 \%$ of children; however, this outcome could have been influenced by use of subsequent treatment. Predictors to achieve CR included older age of the patient at the time of diagnosis, older age of the patient at the time of splenectomy, higher platelet counts in the first month following splenectomy, and a negative correlation with use of prior second-line therapy(ies).

These findings provide robust data in a large cohort of children who have undergone splenectomy for ITP, in particular with regard to the reported findings of an over $90 \% \mathrm{CR} / \mathrm{R}$ rate, with minimal adverse effects in the perioperative period. Age in terms of a predictor for responsiveness is also a novel finding for children. As suggested by the authors, this is perhaps related to the pathophysiology of the disease in teenagers being similar to young adults, who also have improved responsiveness to splenectomy. Although the evidence provided in this study represent novel data in support of consideration of splenectomy as opposed to other forms of second-line therapy, a number of questions remain unanswered. The reason behind the selection of splenectomy and clinical decision-making was not collected, in particular regarding the indication of treatment, e.g., treatment due to bleeding symptoms, disease chronicity, or other HRQoL metrics. Splenectomy also remains the treatment of choice for emergent management of life-threatening bleeding which may be represented by a handful of cases who underwent splenectomy early in the course of their ITP in this cohort. Furthermore, the availability of alternative treatments was variable over the course of cohort enrollment (1997-2017), which possibly influenced selection of 
splenectomy as treatment. The disease phase was also not defined in relation to outcomes. Additional limitations include potential selection bias related to the affiliation with the registry. In addition, the follow-up of the patients was limited to 25 months due to patient retention. It would be important to understand more longterm outcomes (i.e., decades) regarding relapse of disease in adulthood, as well as infectious risk. It may never be possible to conduct randomized trials; however, longitudinal tracking of patients requiring second-line therapy provides the opportunity for indirect comparison. Application of patient-related outcomes in prospective trials may also help to capture factors that matter to patients besides platelet count and help in decision-making.

Despite the unanswered questions and known limitations associated with a registry, the work by Avila et al. provides insight into the long-term outcomes associated with splenectomy in children with ITP. The authors are to be commended for the long-term follow-up and collection of data on a rare group of patients. These data give rise to an important consideration of the safe use of splenectomy to achieve remission in the majority of patients who have undergone this procedure, in spite of the decreasing numbers of patients over time in favor of therapies with presumably fewer life-long side-effects.

\section{References}

1. Aliva ML, Amiri N, Pullenayegum E, Blanchette V, Imbach P, Kühne T Long-term outcomes after splenectomy in children with immune thrombocytopenia: an update on the registry data from the Intercontinental Cooperative ITP Study Group. Haematologica. 2020;105(11): 2682-2685.

2. Freedman J, Blanchette M. Idiopathic thrombocytopenia purpura (ITP): a historical odyssey. Acta Paediatr Suppler. 1998;424:3-6.

3. Stasi R, Newland AC. ITP: a historical perspective. Br J Haematol.
2011;153(4):437-450

4. Werlhof PG. Opera Omnia. Hanover: Helwing, 1775: 748. In: RH Major, ed. Classic descriptions of disease. 3rd ed. Springfield, IL: CC Thomas, 1965

5. Bizzozero G. [Über einer neuen formbestandtheil der blutes und desser role bei der thrombose und der glutgerinnung]. Virchow Arch Pathol Anat Physiol. 1881;90:261-332.

6. Marino F. [Recherches sur les plaquettes du sang. Comptes Rendus des Seances de la Societe de Biologie et de Ses Filiales]. 1905;58:194196.

7. Kaznelson P. [Verschwinden der hämorrhagischen diathese dei einem fall von "essentieller thrombopenie" (Frank) nach milzexstirpation: splenogene thrombolytische purpura]. Wiener Klinische Wochenschrift. 1916;29:1451-1454.

8. Kaznelson P. [Thrombolytische purpura]. Zeitschrift fur Klinische Medizin. 1919;87:133-164.

9. Harrington WJ, Minnich V, Hollingsworth JW, et al. Demonstration of a thrombocytopenic factor in the blood of patients with thrombocytopenic purpura. J Lab Clin Med. 1951;38(1):1-10.

10. George JN, Woolf SH, Raskob GE, et al. Idiopathic thrombocytopenic purpura: a practice guideline developed by explicit methods for The American Society of Hematology. Blood. 1996;88(1):3-40.

11. Eden OB, Lilleyman JS. Guidelines for management of idiopathic thrombocytopenic purpura. The British Paediatric Haematology Group. Arch Dis Child. 1992;67(8):1056-1058.

12. Neunert C, Lim W, Crowther M, et al. The American Society of Hematology 2011 evidence-based practice guideline for immune thrombocytopenia. Blood. 2011;117(16):4190-4207.

13. Neunert C, Terrell DR, Arnold DM, et al. American Society of Hematology 2019 guidelines for immune thrombocytopenia. Blood Adv. 2019;3(23):3829-3866.

14. Reid MM. Chronic idiopathic thrombocytopenia purpura: incidence, treatment, and outcome. Arch Dis Child. 1995;72(2):125 128.

15. Lee EJ, Kueck B. Rituxan in treatment of cold agglutinin disease. Blood. 1998;92(9):3490-3491.

16. Stasi R, Pagano A, Stipa E, et al. Rituximab chimeric anti-CD20 monoclonal antibody treatment for adults with chronic idiopathic thrombocytopenic purpura. Blood. 2001;98(4):952-957.

17. Bennett C, Rogers ZR, Kinnamon DD, et al. Prospective phase 1/2 study of rituximab in childhood and adolescent chronic immune thrombocytopenic purpura. Blood. 2006;107(7):2639-2642.

18. Neunert CE, Rose MJ. Romiplostim for the management of pediatric immune thrombocytopenia: drug development and current practice. Blood Adv. 2019;3(12):1907-1915.

19. Kim TO, Despotovic J, Lambert MP. Eltrombopag for use in children with immune thrombocytopenia. Blood Adv. 2018;2(4):454-461. 